menclature. This last is an area which causes problems for many non-cytogeneticists (and some cytogeneticists!) but the authors give simple definitions of the various terms and employ very clear diagrams depicting the rearrangements. These diagrams are used throughout the book together with photographs of individual chromosomes and this considerably simplifies the task of understanding the geometry of these rearrangements. Part 2 is the real meat of the book : 8 chapters each devoted to a single disease (e.g. CML, ALL) or group of diseases (e.g. malignant lymphomas, solid tumours). The book is very heavily biased towards the haematological malignancies, but this simply reflects the state of our knowledge; $87 \%$ of karyotypes published by 1987 were from haematological disorders. Each chapter contains a brief clinical description of the disease, a discussion of the cytogenetic abnormalities found together with molecular considerations where appropriate, and a separate section on clinico-cytogenetic correlations which is particularly useful for diagnostic laboratories. A vast body of basic material (see below) has been skilfully condensed and is very lucidly presented, with the authors' enthusiasm for the subject coming across clearly, but never being allowed to blur the distinction between fact, hypothesis and opinion. This is also true of the final part, 2 chapters devoted to oncogenes, anti-oncogenes and theories of carcinogenesis, although this is probably the part that suffers most from being overtaken by recent developments.

Throughout the book the authors have been fairly selective in their referencing but many of the references are to the most recent reviews so that it is often possible to delve more deeply with only the minimum of effort.

This book is a landmark in the field of cancer cytogenetics. It is only a fraction of the size and cost of Sandberg's epic tome of 1980 but it is far more accessible to students of genetics and to molecular biologists as well as to the cytogeneticists and clinicians working in the field, among whom it should promote significantly greater understanding.

In contrast to Cancer Cytogenetics Mitelman's Catalog of Chromosome Aberrations in Cancer is a highly specialized book of interest mainly to cytogeneticists and clinicians working very closely with cytogeneticists. For these people, however, this catalog is invaluable. It lists, with references, the karyotypes of all published cases of cancer [other than the $t(9 ; 22)$ of chronic myeloid leukaemia] that Professor Mitelman has been able to find (plus a few unpublished cases). Nearly all of the karyotypes are from direct or short-term culture; cell lines have only been included for a few tumours where other data are particularly scanty. This third edition details 9069 cases from 2156 references, showing the continued rapid expansion of this field since the 5345 cases of the 1985 edition and the 3844 of the 1983 edition. There are several changes in the handling of the material in this edition. The most notable are the addition of sections on double minute chromosomes and homogeneously staining regions, and a section on the molecular analysis of specific breakpoints. Bold type has been used to highlight the chromosome of interest in each karyotype so that it is much easier to scan the lists of complex karyotypes for the information wanted. A certain amount of reorganization of the groupings under morphological diagnosis also facilitates finding what you want, particularly among the solid tumours.

As well as the basic data, Professor Mitelman gives a brief overview of the catalog with interesting tables on the numbers of karyotypes for each disease and the numbers of cases involving each of the 24 chromosomes, clearly showing the non-random nature of chromosome involvement in neoplasia. The catalogue cannot be used to find the overall frequency of abnormalities in any disease as it does not include those cases with a normal karyotype but this is a very minor irritation.

This catalogue represents a vast amount of work and Professor Mitelman is to be congratulated on the frequency with which he continues to update the book. There are remarkably few errors for such a vast enterprise (other than those of the original reporters which are faithfully reproduced) and the changes in the third edition are all welcome. This book is not cheap, but I do not see how anyone in the field of cancer cytogenetics can afford to be without the latest edition of Professor Mitelman's Catalog.

FIONA ROSS

MRC Human Genetics Unit, Western General Hospital, Crewe Road, Edinburgh EH4 $2 X U$

\section{McGraw-Hill Dictionary of Scientific and Technical} Terms. 4th Edition. Editor-in-Chief SYBIL P. PARKer. Maidenhead, Berkshire, UK. 1989. McGraw-Hill Book Co. Ltd. 2088 pages + Appendix. Hard cover. $£ 65.00$. ISBN 0070452709.

This handsome large-format book defines 100100 technical and scientific terms, a number which almost boggles the mind when set against the modest halfmillion words included in the new edition of the Oxford English Dictionary. The obvious implication seems to be that scientists and engineers spend much of their time inventing new terms to make their own disciplines more mysterious and awesome to the outsider-including those embedded in other disciplines. Can we possibly need all this vast array of terms? Or is it that the collectors of the terms to go in each of the 102 categories used in the dictionary vied with each other to amass the greatest number of items?

It is not easy to answer this question. The biologists can claim to have been very modest in listing organisms, since there must be 200000 or more named genera in the literature to choose from. The dictionary 
names a great variety of species or genera, chosen by rules which are not obvious to someone who has only dabbled a little in Taxonomy, as I have. Thus the family Myrmecophagidae gets brief listing but not the great anteater, Myrmecophaga, popular in zoos, nor its two smaller relatives-Tamandua and Cyclopes, which make up this family (however, you will find Myrmecophaga actually illustrated if you know where to look for it, which is under Edentata). The pangolin, or scaly anteater, gets not only a good description but a picture near its name, perhaps because it has a complete family to itself. Plenty of organisms that I have never heard of are listed and many of them pictured, so wild life has received a reasonable amount of attention, even if the rules of choice are unstated and baffling.

Genetics, on the other hand, is clearly underrepresented. First, for the benefit of the enquiring reader, all those organisms which have made major contributions to genetic and medical knowledge should certainly have been listed, with this fact properly recognized in their entries. Obvious examples are Drosophila melanogaster (it is quite insufficient to tell us simply that it is the fruit-fly), the mouse (more space is given to the computer mouse than to the mammal), Escherichia coli and Salmonella typhimurium among quite a few key microorganisms, and Caenorhabditis elegans (not listed), as well as various plants. Also, how did the authors come to misdefine lysogeny as "lysis of bacteria with the liberation of phage particles', and to miss out lambda phage and its key role in genetic engineering entirely? If the meteorologists were allowed to list a variety of obscure winds blowing at certain seasons off certain mountains (among these are ' $n$ 'aschi' in the Persian Gulf, 'touriello' in the Pyrenees, 'Wasatch winds' blowing onto the plains of Utah, and 'tosca'-a south-west wind on Lake Garda in Italy), then the geneticists and molecular biologists should have been given plenty of space for (probably) much more important information. The psychologists, meanwhile, have been able to include words such as 'chrematophobia', meaning an abnormal fear of money-try this word on your bank manager! Other remarkable terms which caught my eye are 'megaloureter' for abnormal enlargement of the ureter, 'megarectum ' for abnormal enlargement of the rectum, msec for a million seconds and (believe it or not) 'megaripple' for a large sand wave.

Important terms which I could not find include: AIDS, electroporation, restriction and modification, restriction fragment, RFLP, PCR (= polymerase chain reaction), selfish genes, selfish DNA, chromosome banding techniques (such as Q-, G-, R- and so on), and even the most frequently used of all molecular genetic techniques - the Southern Blot (let alone the rather more recent Northern Blot). Motoo Kimura's neutral theory of molecular evolution is not there, but I was glad to find the Lyon hypothesis and hybrid dysgenesis listed. Equally surprising is the definition given of the heterogametic sex as "the sex of some species in which the two sex chromosomes differ in gene content or size'. The idea that a species can have a sex is quite a novel one! In computer science I found a few popular terms from the PC literature missing: e.g. DOS, MSDOS, PCDOS, WIMP, WYSIWYG and OS2, and I was particularly disappointed to be unable to find a definition of the word Transputer, which has been appearing in the magazines for some years. Turning to chaos theory, I looked in vain for the Lorenz attractor or Mandelbrot, but fractal and the Koch curve are listed.

The only alternative to this dictionary that I have come across is the Chambers Science and Technology Dictionary, edited by Peter Walker and having a high proportion of its contributors from Edinburgh. Published in 1988, it costs about a quarter as much as the McGraw-Hill Dictionary, contains nearly half as many entries but has no illustrations; and, though a year older, it is clearly more up to date at least to the extent that it includes nearly all those terms which I have listed above as missing from McGraw-Hill. The McGraw-Hill volume has a large format about the same as the journal Nature, is $7 \mathrm{~cm}$ thick, very strongly bound in hard cover and rather heavy (weight $3.22 \mathrm{~kg}$ ). The paper is thin high-quality, presumably India paper, printed with two text columns of $7 \mathrm{~cm}$, leaving an outer column of $5 \mathrm{~cm}$ to accommodate 3000 illustrations in line or half-tone. Many of these illustrations will be found useful, as they include structural chemical formulae, graphs, engineering drawings, pictures of a great variety of organisms, and so on. Thus on the first page we find pictures of aardvark and abalone shell. Notes on pronunciation of each term are included and there is a great variety of useful information in 49 pages of appendix, ending with a biographical listing of important scientists and engineers of the past and (mainly) Nobel Prize winners still alive. Among the living I am glad to see ChingWu Chu (more easily recognized as Paul Chu), who should have got a Nobel Prize for discovering superconductivity at temperatures above $90 \mathrm{k}$ in early 1987. You may like to know that to get your name into the body of the book you only need to have an equation, effect, function, piece of apparatus or zone named after you, which makes a Nobel Prize unnecessary.

On balance, I think this new dictionary deserves a place in all general scientific, military and technical libraries, though not in impecunious biology libraries - which should be able to do well enough with Chambers. My impression, certainly biased by my partial experience of science, is that this new volume has a good many unnecessary terms in some of the disciplines far from my own. Among these the many areas concerned with military apparatus and warfare stand out. But it is very beguiling to turn the pages and find extraordinary definitions such as: 'ropy lava - see pahoehoe' (which I have not done yet). Also, the 
price is reasonable for such a monumental work, and it does define an enormous number of words in unexpected ways. I have no doubt that the majority of these terms are useful in their own disciplines.

ERIC REEVE

Institute of Animal Genetics, University of Edinburgh

\section{Myotonic Dystrophy. 2nd Edition. By PeTER S. HARPER. Harcourt Brace Jovanovich Limited. 384 pages. Price $£ 40.00$. ISBN 0702013641 .}

From many points of view myotonic dystrophy is a fascinating yet tragic condition. It is inherited as an autosomal dominant trait and extensive families with the condition are not uncommon. The manifestations are protean and include not only myotonia, which gives the condition its name and refers to a delayed relaxation after a muscle contracts, but also muscle weakness which affects mainly the facial as well as the more distal limb musculature, smooth and cardiac muscle involvement, some degree of mental deterioration, various endocrine abnormalities, cataracts, premature baldness and various skin and skeletal abnormalities. However, a gene carrier may sometimes demonstrate no more than very mild myotonia or symptomless cataracts and the demonstration of these defects in the past provided tests for presymptomatic diagnosis.
However the gene locus has been located on the long arm of chromosome 19 and several closely linked DNA markers (muscle creatine kinase $\mathrm{MM}$, apolipoproteins $\mathrm{Cl}$ and $\mathrm{C2}$ ) are now proving valuable for presymptomatic as well as prenatal diagnosis. But the gene itself has not yet been isolated, and the basic biochemical defect still remains unknown. Though drugs can be useful in controlling disabling myotonia there is as yet no effective treatment. Furthermore, several fascinating aspects still remain unexplained. These include the wide range in age at onset, the fact that some individuals may remain only slightly affected throughout their lives whereas others may be severely affected even from birth, particularly in the case of infants born to affected mothers. Finally, anticipation, which refers to progressively earlier onset and greater severity in successive generations, now seems likely to be a true phenomenon and not, as many once thought, merely a reflection of biases in sampling affected families.

All these aspects of the condition are reviewed in detail in this new edition, which is a well written, well referenced, up-to-date, and scholarly text, the author himself being a world authority on the disorder. It has no competitors and can be highly recommended to anyone interested in this fascinating condition.

ALAN EMERY Medical School, University of Edinburgh 